\title{
Gravitational waves from accreting systems
}

\author{
Rosa Poggiani* $\dagger$ \\ Università di Pisa and Istituto Nazionale di Fisica Nucleare, Sezione di Pisa \\ E-mail: rosa.poggiani@unipi.it
}

\begin{abstract}
The recent discovery of gravitational waves has opened a new window in astronomy. Accreting systems are very common in astrophysics and are multifreqeuncy sources, since their emission spans the whole electromagnetic spectrum. Accreting systems in astronomy are also multimessenger sources, since they can emit gravitational waves via a variety of physical mechanisms and because of their intrinsical binary nature. This paper presents a review of the gravitational wave emission of accreting systems at all scales, from cataclysmic variables to active galactic nuclei, showing that the emission occurs over the whole gravitational spectrum.
\end{abstract}

Multifrequency Behaviour of High Energy Cosmic Sources - XIII - MULTIF2019

3-8 June 2019

Palermo, Italy

\footnotetext{
*Speaker.

${ }^{\dagger}$ Corresponding author
} 


\section{Introduction}

The recent observations of gravitational waves from binary black hole mergers and a binary neutron star merger has opened a new observational window in astronomy [3], [4], [6], [7], [8], [9], [15]. The spectrum of gravitational waves from different sources reported in Fig. 1 covers several decades in frequency, demanding for different observational strategies. The compact binary coalescences detected with the ground based interferometers Advanced LIGO and Advanced Virgo belong to the high frequency region, ranging from a few $\mathrm{Hz}$ to some $\mathrm{kHz}$, where also the continuous emission from pulsars and the supernova collapse bursts are expected. The sensitivity of ground based interferometers below a few $\mathrm{Hz}$ is limited by seismic noise, requiring space based interferometers, the future instruments LISA [22] and TianQin [63]. Space based interferometers are designed to detect resolved binaries, the merger of massive binary black holes and the final stage of the merger of stellar black holes, with the possibility to trigger ground based interferometers to perform multifrequency gravitational wave astronomy [74]. The very low frequency region below $10^{-5} \mathrm{~Hz}$ includes the emission of supermassive binaries, the astrophysical stochastic background of coalescing supermassive black hole binaries and the cosmological background of gravitational waves from phase transition in the early universe [55], [56]. The emission in this region relies on pulsar timing techniques [65], [52], [62] [53].

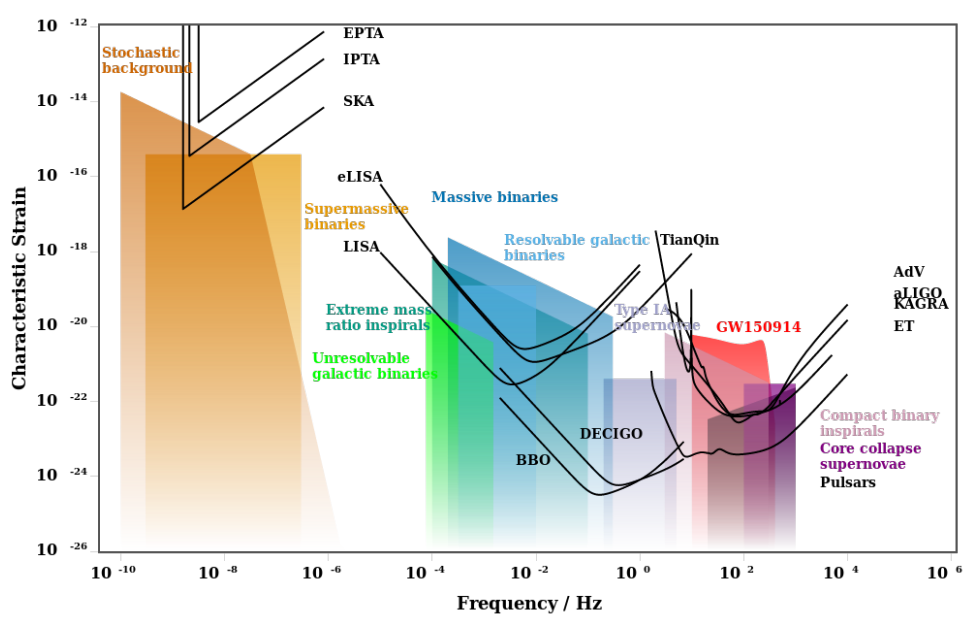

Figure 1: The gravitational wave spectrum; credits: http://gwplotter.com/

Accreting systems appear in the list of binaries discussed above. Accretion appears at all scales in astrophysics, from young stellar objects to white dwarfs to neutron stars to black holes of all masses [47]. The standard model of accretion has been proposed by [75].

In this paper, the gravitational wave emission from accreting sources will be discussed from two points of view, the emission related to the intrinsic binary nature of systems and the emission produced by physical processes related to accretion, discussing different sources: cataclysmic variables, accreting neutron stars, accreting black holes in the Galactic Center and in active galactic nuclei. The variety of processes involved in accreting systems covers the whole electromagnetic spectrum, requiring the combination of different observational techniques. 


\section{Gravitational Waves from Binary Systems}

Accreting systems are binary systems, where gravitational wave emissions occurs at twice the orbital frequency and harmonics. When the orbital eccentricity is negligible, the contribution of harmonics is negligible and the gravitational wave characteristic amplitude is given by [78]:

$$
h=8.7 \times 10^{-21}\left(\frac{\mu}{M_{\odot}}\right)\left(\frac{M}{M_{\odot}}\right)^{\frac{2}{3}}\left(\frac{100 p c}{r}\right)\left(\frac{f}{10^{-3} H z}\right)^{\frac{2}{3}}
$$

where $M=M_{1}+M_{2}, \mu=\frac{M_{1} M_{2}}{M_{1}+M_{2}}, M_{1}, M_{2}$ are the masses of the primary and secondary components, $r$ is the distance of the system and $f$ the gravitational wave frequency.

\section{Cataclysmic Variables}

Cataclysmic variables are binaries composed by a white dwarf and a secondary star. The gravitational wave emission of cataclysmic variables has been estimated by [26] and [64], for about 160 objects each, and by [70], for about 500 systems. The catalogue by Ritter and Kolb [72], [73] ${ }^{1}$, version 7.23, contains more than 1400 systems. The orbital period is known for the majority of the cataclysmic variable, but the masses of the primary and of the secondary mass are known for about $10 \%$ of objects. The missing masses can be estimated using the approach by [77], [64], as discussed by [70]. Before the advent of Gaia, the distances of cataclysmic variables have been measured with different methods. The compilation of distances used by [70] combine distances estimated using the parallax, the nova expansion parallax and the Period-Luminosity-Colour relation by [18], [19].

The gravitational strain estimated by [70] for an observation time of two years is shown in Fig. 2. The solid curve is the sky averaged design sensitivity of LISA [24], with an arm length of $2.5 \times$ $10^{6} \mathrm{~km}$, an acceleration noise extrapolated from LISA Pathfinder performances, a binary confusion noise estimated by [22]. The dotted curve is the sensitivity of the original LISA design, with $5 \times 10^{6}$ $\mathrm{km}$ arm length [60]. The dashed curve is the confusion noise, a background of astrophysical origin from unresolved binary systems [51], [30]. The most promising sources are the very short period AM CVn systems.

\section{Accreting Neutron Stars}

The gravitational emission from accreting neutron stars has been discussed by [86], who suggested that accreting neutron stars with weak magnetic fields acquiring angular momentum can become unstable. When the growth time of instability is balanced by the viscous damping time, the gravitational strain is given by:

$$
h=3 \times 10^{-27}\left(\frac{1 \mathrm{kHz}}{\mathrm{mf}}\right)^{1 / 2}\left(\frac{F_{x}}{10^{-8} \operatorname{erg~s}^{-1} \mathrm{~cm}^{-2}}\right)
$$

where $m$ is the mode number, $F_{x}$ the time averaged X-ray flux, $f$ the frequency of the monochromatic gravitational wave. The accreting neutron star should undergo a non axial distortion, with

\footnotetext{
${ }^{1}$ http://wwwmpa.mpa-garching.mpg.de/RKcat/
} 


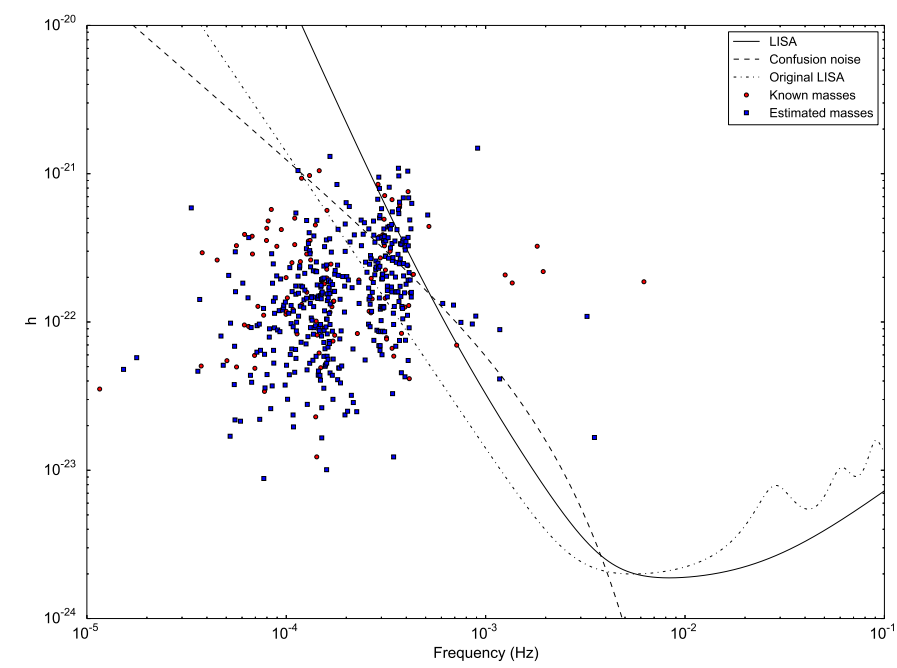

Figure 2: Gravitational wave emission of cataclysmic variables with known masses (red circles) and estimated masses (blue squares), adapted from [70]; the solid curve is the instrumental sensitivity of the new design LISA interferometer [24], the dotted line of the original LISA [60], the dashed line is the binary confusion noise [60]

a modulation of the X-ray emission occurring at the gravitational wave frequency, of the order of some hundreds $\mathrm{Hz}$ [86]. The abundance of the spin frequencies of neutron stars around $300 \mathrm{~Hz}$ suggested that the angular momentum from the accretion was converted into gravitational waves [31]. The estimated strain of accreting neutron stars is of the order of $10^{-26}$. The elastic deformation of the crust due to density perturbations caused by electron capture reactions has been discussed by [80], who estimated the quadrupole moment required to balance the torque by accretion, about $10^{37}-10^{38} \mathrm{~g} \mathrm{~cm}^{2}$ for accretion rates in the range $10^{-10}-10^{-8} \mathrm{M}_{\odot} \mathrm{yr}^{-1}$. The conditions for a steady gravitational wave emission have been discussed by [87], who suggested that stable equilibrium will occur when the superfluid transition temperature of hyperons is smaller than $\sim 2 \times 10^{9} \mathrm{~K}$. Gravitational wave emission can drive an instability in the r-modes of young rotating neutron stars [68], producing angular momentum loss and a spin frequency decrease. An instability in rotating neutron stars, driven by gravitational radiation reaction and affecting the r-modes, sets an upper limit on the spin frequency of young neutron stars [23]. A rapidly rotating neutron star can potentially convert about $0.01 \mathrm{M}_{\odot}$ into gravitational radiation. Magnetically confined mountains on accreting neutron stars could produce detectable gravitational waves [85], [50]. The work by [89] suggested that a few systems accreting around the Eddington rate can be potentially detected by advanced interferometers. The statistics of detectable systems increases if their spin and orbital parameters are known from electromagnetic measurements, since data analysis algorithms rely on the folding of long time records [89]. A detailed investigation of the evolution of the low-mass $\mathrm{X}$ ray binaryies and the relation with gravitational wave emission has been presented by [32]. The stage of common envelope in accreting neutron stars involves gravitational wave emission. Neutron stars accrete at a fraction of the Bondi-Hoyle-Lyttleton rate, but they could be able to accrete at hypercritical rates, producing strains exceeding those of Sco X-1 [54].

The knowledge of the spin and orbital periods is of great importance for the computational cost 
of the searches for gravitational wave emission of accreting neutron stars. In the catalogue by [61], spin and orbital periods are known for less than $15 \%$ and less than $50 \%$ of systems, respectively. Precision ephemerides for Low Mass X-Ray Binaries are measured using optical spectroscopy and have been recently determined for Sco X-1 [45], [88] and Cyg X-2 [71].

The archives of X-ray observatories allow searches for periodicities, that include orbital and spin periods, and also superorbital periods and outburst recurrence times. Systematic search of periodicities in X-ray binaries have been presented by [90] (whole RXTE ASM archive). Targeted search of periodicities of X-ray binaries have been presented by [38], [39] (Swift BAT archive), [57] (RXTE ASM archive) and by [69] (Swift BAT and RXTE ASM archives).

Sco X-1 is a potential bright source of continuous gravitational waves and has been investigated by the initial and advanced interferometers. The upper limit by initial LIGO has been set using semicoherent techniques [1]. During the observing runs $\mathrm{O} 1$ and $\mathrm{O} 2$ of the Advanced LIGO detectors upper limits have been set using model based cross-correlation searches [10] and Hidden Markov Models [11], [14]. Directional unmodeled searches for persistent gravitational waves from Sco X-1 have been performed with the initial LIGO [2] and the Advanced LIGO O1, O2 runs [12], [13]. For reference, the best upper limits on Sco X-1 emission estimated in the searches are of the order of $10^{-25}$.

\section{Neutron Star-Black Hole Systems}

No Neutron Star-Black Hole merger has been detected to date. The upper limit on the merger rate estimated with Advanced LIGO is $3600 \mathrm{Gpc}^{-3} \mathrm{yr}^{-1}$ [5]. This class of events can involve accreting systems. Some X-ray binaries have been proposed as candidate progenitors of mergers involving neutron star and black holes [29]: Cyg X-1, Cyg X-3, MWC 656, P13 in NGC 7793. These sources contain a black hole with a mass in the range from 5 to $15 \mathrm{M}_{\odot}$ and a secondary star with a mass in the range from 7 to $20 \mathrm{M}_{\odot}$ [29]. The four systems have allowed estimations of the merger rates: $0.028-0.4 \mathrm{yr}^{-1}$ for Cyg X-1 [27], 0.09-0.15 $\mathrm{yr}^{-1}$ for Cyg X-3 [28], 0-0.187 $\mathrm{yr}^{-1}$ for MWC 656 [49], up to 0.1-0.6 $\mathrm{yr}^{-1}$ for P13 [29]. MWC 656 is a peculiar source, since it is the first detected Be/Black hole system [34], [67], [42], [20].

\section{Galactic Center}

The observations of the orbits of the $\mathrm{S}$-stars provides the evidence for $\mathrm{Sgr} \mathrm{A}^{*}$, a supermassive black hole with a mass of about $4 \times 10^{6} \mathrm{M}_{\odot}$ at the center of our Galaxy [43]. Searches for persistent gravitational waves from the Galactic center have been performed with the initial [2] and advanced LIGO interferometers [12], [13], setting upper limits of the order of $10^{-25}$. The S-stars are examples of Extreme Mass Ratio Inspiral (EMRI) systems that undergo tidal disruption during the inspiral stgae, with a gravitational emission in the range that is investigated with Pulsar Timing techniques [33]. The properties of $37 \mathrm{~S}$-stars orbiting Sgr A* have been presented by [46]. The gravitational waveforms of S-stars orbiting $\mathrm{Sgr} \mathrm{A}^{*}$ [33] have frequencies in the nanoHz range and amplitudes in the range $10^{-20}-10^{-19}$, some orders of magnitude below the sensitivity of current and planned Pulsar Timing Arrays. 
Other gravitational wave emitters could by detected with the space based interferometer LISA. The gravitational emission from general objects orbiting Sgr $\mathrm{A}^{*}$ in circular orbits has been discussed by [48], who showed that compact objects with masses larger than $10^{-4} \mathrm{M}_{\odot}$, main sequence stars with mass lower than $2.5 \mathrm{M}_{\odot}$ can be detected with one year observation. Stellar mass black holes with masses of about $40 \mathrm{M}_{\odot}$ inspiralling around $\mathrm{Sgr} \mathrm{A}^{*}$ could also be detected by LISA [44].

\section{Active Galactic Nuclei}

Blazars are active galactic nuclei powered by accretion onto one or two supermassive black holes, exhibiting variability over the whole electromagnetic spectrum [79], with time scales ranging from minutes to decades. Blazars have radio jets that can show an apparent superluminal motion. The presence of a pair of black holes, of jets and the variability at small scale can produce gravitational wave emission.

Supermassive black hole binaries in active galactic nuclei can emit gravitational waves due to their orbital motion. The presence of a black hole pair is inferred looking at the periodicities in the light curve of the source (see e.g. [37]). OJ 287 has been modeled as a binary black hole system including precession [76], [58], [81], [82], [83]. The light curve of OJ 287 reported in Fig. 3 extends for more than one century and shows two peaked outbursts with a recurrence time of about 12 years, associated to the orbital motion of the black holes. The timing the 2007 outburst demonstrated the energy loss via gravitational radiation [84], that include reaction effects and can allow to test the no-hair theorem [41].

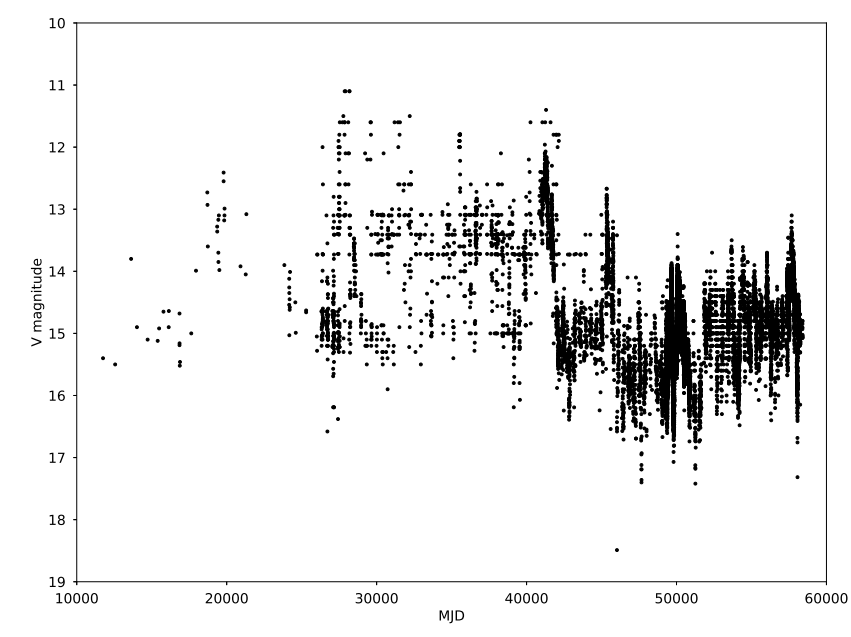

Figure 3: V band light curve of OJ 287 (data from http://altamira.asu.cas.cz/iblwg/data/oj287/ and AAVSO)

Since the detected periodicities of blazars range from years to decades, the gravitational radiation is emitted at about $10^{-9}-10^{-8} \mathrm{~Hz}$, the domain of pulsar timing. Upper limits on the gravitational wave emission from supemassive black hole binaries [16] and OJ 287 [36] have been set. 
Gravitational emission can be caused by the ejection of jet superluminal components and the precession of accretion disks [66], [21]. The superluminal emission in blazars and other objects, such as microquasars, could be caused by the combination of the accretion disk precession induced by a central Kerr black hole and the fragmentation of the tilted disk, dynamically driven by the Bardeen-Petterson effect [25]. Gravitational wave bursts can occur during the initial acceleration of the jet, with a frequency spectrum in the region of the space based interferometer LISA [66], [21].

Blazar flares can last for months, but often variability at short time scales occurs. An example is the gamma variability at the scale of minutes observed in PKS 2155-304 by HESS in July 2006 [17]. Gravitational wave emission associated to the flare could occur at the same time of electromagnetic emission [40] and could be fast enough to be detectable with ground based interferometers. The physical mechanisms of fast flares [40] could be the fragmentation of accretion disk for a blazar hosting a single supermassive black hole [59], the impact from the accretion disk of the secondary black hole in blazars hosting pairs of supermassive black holes [82], dynamical friction from satellites infalling onto a coplanar accretion disk [35].

\section{Conclusions}

Several accreting systems are candidate gravitational sources, either as binaries or because of physical processes: cataclysmic variables, X-ray binaries, active galactic nuclei. Their emission span the whole gravitational spectrum, requiring the combination of different observational techniques: ground based interferometry, space based interferometry, pulsar timing.

\section{References}

[1] J. Aasi et al., PRD 91 (2015) 062008.

[2] J. Abadie et al, PRL 107 (2011) 271102.

[3] B. P. Abbott et al., PRL 116 (2016) 061102.

[4] B. P. Abbott et al., PRL 116 (2016) 241103.

[5] B. P. Abbott et al., ApJL 832 (2016) L21,

[6] B. P. Abbott et al., PRL 118 (2017) 221101.

[7] B. P. Abbott et al., ApJ 851 (2017) L35.

[8] B. P. Abbott et al., PRL 119 (2017) 141101.

[9] B. P. Abbott et al., PRL 119 (2017) 161101.

[10] B. P. Abbott et al., ApJ 847 (2017) 47.

[11] B. P. Abbott et al., PRD 95 (2017) 122003.

[12] B. P. Abbott et al., PRL 118 (2017) 121102.

[13] B. P. Abbott et al., PRD 100 (2019) 062001.

[14] B. P. Abbott et al., PRD 100 (2019) 122002. 
[15] B. P. Abbott et al., $P R X 9$ (2019) 031040.

[16] K. Aggarwal et al., ApJ 880 (2019) 116.

[17] F. Aharonian et al., ApJ 644 (2007) L71.

[18] T. Ak et al., NewA 12 (2007) 446.

[19] T. Ak et al., NewA 13 (2008) 133.

[20] B. Aleksic et al., A\&A 576 (2015) 36.

[21] W. D. Alfonso et al., AN 336 (2015) 815.

[22] P. Amaro-Seoane et al., arXiv:1702.00786.

[23] N. Andersson et al., ApJ 510 (1999) 846.

[24] S. Babak et al., PRD 95 (2017) 103012.

[25] J. M. Bardeen and J. A. Petterson, ApJ 195 (1975) L65.

[26] F. Barone et al., GRG 24 (1992) 323.

[27] K. Belczynski et al., ApJ 742 (2011) L2.

[28] K. Belczynski et al., ApJ 764 (2013) 96.

[29] B. Beldycki and K. Belczynski, AcA 66 (2016) 347.

[30] P. L. Bender and D. Hils, CQG 14 (1997) 1439.

[31] L. Bildsten, ApJ 501 (1998) L89.

[32] G. S. Bisnovatyi-Kogan, Astroph 32 (1990) 176.

[33] B.-G. Cai et al., Comm. Theor. Phys. 70 (2018) 735.

[34] J. Casares et al., Nat 505 (2014) 378.

[35] P. Chang et al., ApJ 684 (2008) 236.

[36] J.-W. Chen and Y. Zhang, MNRAS 481 (2018) 2249.

[37] S. Ciprini et al., in Volume 288 - Accretion Processes in Cosmic Sources (APCS2016) - ACCRETION ONTO WHITE DWARFS, NEUTRON STARS \& BLACK HOLES, PoS(APCS2016)044.

[38] R. Corbet et al., PThPS 169 (2007) 200.

[39] R. H. D. Corbet and H. A. Krimm, ApJ 778 (2013) 45.

[40] S. Desai et al., CQG 25 (2008) 184024.

[41] L. Dey et al., ApJ 866 (2018) 11.

[42] S. A. Dzib et al., $A \& A \mathbf{5 8 0}$ (2015) 6.

[43] A. Eckart et al., Found. Phys. 47 (2017) 553.

[44] R. Emami and A. Loeb, arXiv:1903.02579v3.

[45] D. K. Galloway et al., ApJ 781 (2014) 14.

[46] S. Gillessen et al., ApJ 837 (2017) 30. 
[47] F. Giovannelli, in Volume 342 - Accretion Processes in Cosmic Sources - II (APCS2018), PoS(GOLDEN 2017)001.

[48] E. Gourgoulhon et al., $A \& A 627$ (2019) A92.

[49] M. Grudzinska et al., MNRAS 452 (2015) 2773.

[50] B. Haskell et al., MNRAS 450 (2015) 2302.

[51] D. Hils et al., ApJ 360 (1990) 65.

[52] G. Hobbs et al., CQG 27 (2010) 084013.

[53] G. Hobbs and S. Dai, NSR 4 (2017) 707.

[54] A. M. Holgado et al., ApJ 857 (2018) 38.

[55] F. Jenet et al., ApJ 625 (2005) L123.

[56] F. Jenet et al., ApJ 653 (2006) 1571.

[57] M. M. Kotze and P. A. Charles, MNRAS 420 (2012) 1575.

[58] H. J. Lehto and M. J. Valtonen, ApJ 460 (1996) 207.

[59] Y. Levin et al., astro-ph/0307084.

[60] LISA Study Team, 1998, in LISA Pre-Phase A Report. 2nd Edition, Publication MPQ-233 (1998) Max-Plank Institute for Quantum Optics, Garching.

[61] Q. Z. Liu et al., $A \& A 469$ (2007) 807.

[62] A. Lommen, RPP 78 (2015) 124901.

[63] J. Luo et al., CQG 32 (2016) 035010.

[64] M. T. Meliani et al., $A \& A 358$ (2000) 417.

[65] C. J. Moore et al., CQG 32 (2015) 055004.

[66] H. J. Mosquera Cuesta et al., OAJ 4 (2011) 98.

[67] P. Murar-Adrover et al, ApJ 829 (2016) 101.

[68] B. J. Owen et al., $P R$ D 58 (1998) 084020.

[69] R. Poggiani, in Volume 233 - Swift: 10 Years of Discovery, (SWIFT 10), PoS(SWIFT 10)157.

[70] R. Poggiani, in Volume 315 - The Golden Age of Cataclysmic Variables and Related Objects IV (GOLDEN 2017), PoS(GOLDEN 2017)008.

[71] S. S. Premachandra et al., ApJ 823 (2016) 106.

[72] H. Ritter and U. Kolb, $A \& A 404$ (2003) 301.

[73] H. Ritter and U. Kolb, Acta Polytech. CTU Proc. 2 (2015) 21.

[74] A. Sesana, PRL 116 (2016) 231102.

[75] Ni. I. Shakura and R. A. Sunyaev, $A \& A 24$ (1973) 337.

[76] A. Sillanpää et al., ApJ 325 (1988) 628.

[77] D. A. Smith and V. S. Dhillon, MNRAS 301 (1998) 767. 
[78] K. S. Thorne, in Three Hundreds Years of Gravitation (1987) 330, Cambridge University Press, Cambridge, eds. S. Hawking and W. Israel.

[79] M. Urry and P. Padovani, PASP 107 (1995) 803.

[80] G. Ushomirsky et al., MNRAS 319 (2000) 902.

[81] M. J. Valtonen et al., ApJL 643 (2005) L9.

[82] M. J. Valtonen et al., ApJ 646 (2006) 36.

[83] M. J. Valtonen, ApJ 657 (2007) 1074.

[84] M. J. Valtonen et al., Nat 452 (2008) 851.

[85] M. Vigelius and A. Melatos, MNRAS 395 (2009) 1972.

[86] R. V. Wagoner, ApJ 278 (1984) 345.

[87] R. V. Wagoner, ApJ 578 (2002) L63.

[88] L. Wang et al., MNRAS 478 (2018) 5174.

[89] A. L. Watts et al., MNRAS 389 (2008) 839.

[90] L. Wen et al., ApJS 163 (2006) 372. 